\title{
The Biological Effects of Sex Hormones on Rabbit Articular Chondrocytes from Different Genders
}

\author{
Shwu Jen Chang, ${ }^{1}$ Shyh Ming Kuo, ${ }^{1}$ Yen Ting Lin, ${ }^{1}$ and Shan-Wei Yang ${ }^{2}$ \\ ${ }^{1}$ Department of Biomedical Engineering, I-Shou University, Kaohsiung 81346, Taiwan \\ ${ }^{2}$ Department of Orthopedics, Kaohsiung Veterans General Hospital, 386 Ta-Chung 1st Road, Kaohsiung 81300, Taiwan \\ Correspondence should be addressed to Shan-Wei Yang; yangshanwei@yahoo.com.tw
}

Received 17 February 2014; Revised 12 May 2014; Accepted 12 May 2014; Published 3 June 2014

Academic Editor: Magali Cucchiarini

Copyright (c) 2014 Shwu Jen Chang et al. This is an open access article distributed under the Creative Commons Attribution License, which permits unrestricted use, distribution, and reproduction in any medium, provided the original work is properly cited.

\begin{abstract}
The aim of this study was to investigate the biological effects of sex hormones (17 $\beta$-estradiol and testosterone) on rabbit articular chondrocytes from different genders. We cultured primary rabbit articular chondrocytes from both genders with varying concentration of sex hormones. We evaluate cell proliferation and biochemical functions by MTT and GAG assay. The chondrocyte function and phenotypes were analyzed by mRNA level using RT-PCR. Immunocytochemical staining was also used to evaluate the generation of collagen-II. This study demonstrated that $17 \beta$-estradiol had greater positive regulation on the biological function and gene expressions of articular chondrocytes than testosterone, with the optimal concentrations of $10^{-6}$ and $10^{-7} \mathrm{M}$, particularly for female chondrocytes.
\end{abstract}

\section{Introduction}

Osteoarthritis $(\mathrm{OA})$ is a degenerative joint disease that causes cartilage erosion, synovial inflammation, and osteophyte formation. Furthermore, articular cartilage has a limited self-healing potential after injury or degeneration, and the healing and repair of injured cartilage remains a difficult clinical problem. The incidence of OA increases with age in both genders, but there is a higher prevalence in females after the age of 50 years $[1,2]$. Sex hormones, especially estrogens and androgens, have been considered to be possible factors for the predisposition to OA, and the depletion or altered metabolism of these hormones has been regarded as a risk for OA [3-5]. However, the correlation between sex hormones and OA is inconsistent. The effective doses on articular cartilage are inconclusive, and varying results have been reported. $\mathrm{Ng}$ et al. reported that physiological doses of estradiol did not alter proteoglycan synthesis for fish chondrocytes [6]. Other studies stated that supraphysiological estradiol protects articular chondrocytes against reactive oxygen species-induced damage, and antioxidative effects are observed after repeated physiological estradiol treatment [7]. Moreover, a decrease of collagen II degradation products in the urine has been observed following estrogen application in ovariectomized rats [8]. Claassen et al. showed a statistically significant decrease in the GAG content in miniature pigs with an experimentally induced estrogen deficiency in comparison with nontreated animals [9]. Furthermore, collagen II synthesis was found to be increased when chondrocytes from the rib growth zone of female rats were incubated with estradiol, supporting the hypothesis that estradiol deficiency in postmenopausal women might be associated with a higher incidence of OA [10]. In another study, Irie et al. reported that castration led to an increase in apoptosis and a decrease in the proliferation of chondrocytes in the growth plate; in addition, fewer chondrocytes were observed in the castrated rabbits than in normal animals of the same age [11].

To evaluate the effect of sex hormones (estradiol and testosterone) on the physiological regulation of chondrocytes, we designed experiments to investigate the biological effects of sex hormones on the rabbit chondrocytes from different genders. OA is characterized by the loss of collagens and proteoglycans in the extracellular matrix (ECM) of articular cartilage. Matrix metalloproteinases (MMPs) degrade several ECM molecules, including cartilage proteoglycan and collagen II. Tissue inhibitor of metalloproteinase 
(TIMP) is a glycoprotein that inhibits all MMPs on a $1: 1$ basis by forming high-affinity complexes. Some studies have suggested that there may be an overexpression of MMPs relative to the TIMPs in OA joints [12]. In addition to Col-I, Col-II, and aggrecan gene expression, we additionally addressed whether MMP-3 and TIMP-3 gene expression was affected differently in the genders. We cultured primary rabbit articular chondrocytes following incubations with sex hormones and used MTT assay, GAG assay, and real-time reverse transcription polymerase chain reactions (RT-PCR) to analyze the function and phenotype of the chondrocytes. Immunocytochemical staining was also performed at the end of culture.

\section{Materials and Methods}

2.1. Materials. $\beta$-Estradiol was purchased from Sigma (USA). Testosterone was purchased from Fluka (Switzerland). All of the other chemicals used in this study were of analytical reagent grade and were used without further purification.

2.2. Chondrocyte Isolation. Chondrocytes were isolated from the articular cartilage of six male and six female Japanese white rabbits (12 weeks of age). All procedures conformed to the guidelines of the Institute of Animal Care and Use Committee of I-Shou University. After aseptic preparation, the articular cartilage from the knee joint of the hind leg was dissected using a surgical blade and minced into $1 \mathrm{~mm}^{3}$ samples. These samples were rinsed twice with phosphatebuffered saline (PBS) and digested with protease $(2 \mathrm{mg} / \mathrm{mL}$, Sigma) for 2 hours followed by $0.2 \%$ collagenase II (Sigma) in PBS at $37^{\circ} \mathrm{C}$ for $3 \mathrm{~h}$. The digested cell suspension was centrifuged at 1,500 rpm for $5 \mathrm{~min}$ to pellet the chondrocytes. The cell pellet was resuspended (in a $75 \mathrm{~cm}^{2}$ flask) in Ham's F12 medium (GIBCO) supplemented with 10\% fetal bovine serum and antibiotics (200 U/mL penicillin/streptomycin), and the cells were cultured in an incubator at $37^{\circ} \mathrm{C}$ and $5 \%$ $\mathrm{CO}_{2}$.

2.3. Cell Culture and Sex Hormone Incubation. Primary rabbit articular chondrocytes from both genders were cultured separately. The second passage of chondrocytes was used, and the cells at a final density of $5 \times 10^{4}$ cells $/ \mathrm{mL}$ were incubated with various concentrations of sex hormones $\left(10^{-5}, 10^{-6}\right.$, $10^{-7}, 10^{-8}$, and $10^{-9} \mathrm{M}$ ), either $\beta$-estradiol or testosterone, for 3 days. An untreated culture was used as the control.

2.4. MTT Assay. Cell viability was determined using MTT assay. After exposure to sex hormones for 3 days, MTT solution was added, and the cells were incubated for another $3 \mathrm{~h}$. The supernatant was removed from the precipitated reaction products, and the precipitates were dissolved in dimethylsulfoxide (DMSO). The absorbance was measured using a multiplate reader (Thermo Scientific) at $570 \mathrm{~nm}$.

2.5. GAG Assay. A commercially available GAG assay kit (Blyscan Assay Kit) was used to determine the total amount of GAG in the chondrocyte cultures. Briefly, culture medium samples were harvested and reacted with the Blyscan dye reagent that was composed of 1,9-dimethylmethylene blue (DMMB) for $30 \mathrm{~min}$, and the unbound dye solution was removed by centrifugation. The bound dye was released from the insoluble GAG-dye complex using a dissociation reagent and quantified spectrophotometrically by measuring the absorbance at $650 \mathrm{~nm}$. The total amount of GAG was determined using a standard curve that was generated using chondroitin 4-sulfate as a standard.

2.6. Real-Time PCR Analysis. The expression levels of collagen I (Col-I), collagen II (Col-II), aggrecan, TIMP-3, and MMP-3 were evaluated at the mRNA level using RTPCR. Briefly, the total RNA was extracted using a Microto-Midi Total RNA Purification System according to the manufacturer's protocol. A total of $5 \mu \mathrm{g}$ of total RNA was used to synthesize cDNA using the SuperScript III FirstStrand Synthesis System (Invitrogen). The cDNA was amplified using SYBR Green PCR reagents, and the RT-PCR reactions were conducted using the StepOne Real-Time PCR System (Applied Biosystems). An initial activation step was performed at $95^{\circ} \mathrm{C}$ for $30 \mathrm{sec}$, followed by 40 cycles at $95^{\circ} \mathrm{C}$ for $3 \mathrm{sec}$ and $60^{\circ} \mathrm{C}$ for $30 \mathrm{sec}$. All of the reactions were performed in duplicate, followed by a meltingcurve analysis for each RT-PCR run. The expression levels were calculated using the comparative CT method [13]. The following primer sequences were used: aggrecan, $5^{\prime}$ ATCTACCGCTGTGAGGTGAT-3 ${ }^{\prime}$ (forward) and $5^{\prime}$-CTCCTGGAAGGTGAACTTCT-3' (reverse); collagen type I, $5^{\prime}$ ACGCATGAAGGCAAGTTGGGTAG-3' (forward) and $5^{\prime}$ AGCAGTGGTTACTACTGGATCGATC-3' (reverse); collagen type II, 5'-AAGAGCGGTGACTACTGGAT-3' (forward) and $5^{\prime}$-ACGCTGTTCTTGCAGTGGTA-3' (reverse); TIMP-3, 5' -TCTGCAACTCCGACATCGTG-3' (forward) and $5^{\prime}$-CGGATGCAGGCGTAGTGTT-3' (reverse); MMP3, $5^{\prime}$-GGCCATCTCTTCCTTCAG-3' (forward) and $5^{\prime}$ GTCACTTTCTTTGCATTTGG-3' (reverse); GAPDH, $5^{\prime}$ GTCAAGGCTGAGAACGGGAA-3' (forward) and $5^{\prime}$ GCTTCACCACCTTCTTGATG-3' (reverse). GAPDH was used as a housekeeping gene [14].

2.7. Immunocytochemistry of Cultured Articular Chondrocytes. For immunocytochemistry, cells were washed twice with PBS and fixed with $4 \%$ paraformaldehyde (Sigma) in PBS ( $\mathrm{pH}$ 7.4) for $20 \mathrm{~min}$ at room temperature. After washing 3 times with PBS, the cells were permeabilized with $0.25 \%$ Triton X-100 (Sigma) in PBS for $20 \mathrm{~min}$ at room temperature and then blocked with $0.5 \%$ fetal bovine serum in PBS for 1 hour. The cells were then incubated overnight at $4^{\circ} \mathrm{C}$ with primary antibodies against Col-I (COL1A, sc-59772, 1:400) and Col-II (COL2A1, sc-52658, 1:500) (both from Santa Cruz Biotechnology, Santa Cruz, CA) [15]. The next day, the cells were washed 3 times with PBS and incubated with the corresponding secondary antibody in the dark for $1 \mathrm{~h}$ at room temperature; goat anti-mouse IgG-R (sc2092, 1:400) was used for Col-I and goat anti-mouse IgGFITC (sc-2010, 1:400) was used for Col-II (both from Santa Cruz Biotechnology). The cells were then stained with 4',6-diamidino-2-phenylindole (DAPI; Invitrogen, USA) to 


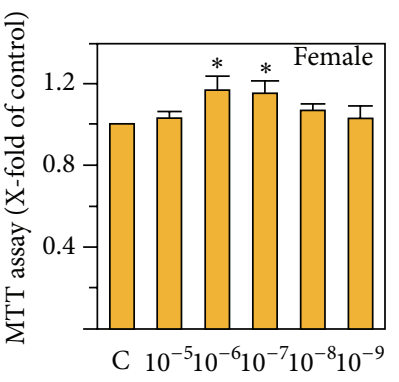

Concentration of estradiol (M)

(a)

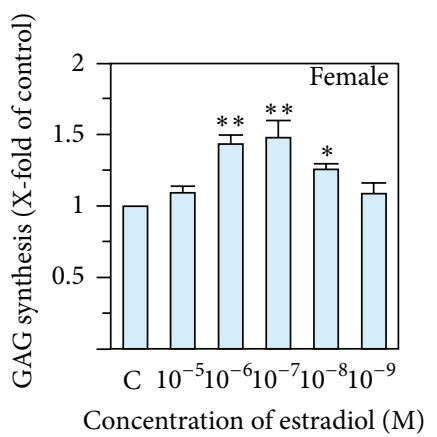

(e)

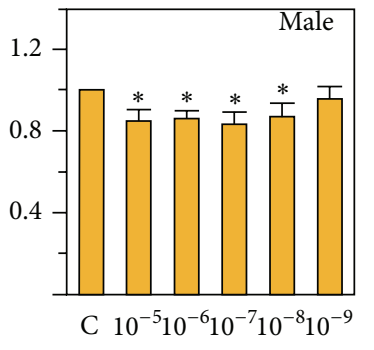

Concentration of estradiol (M)

(b)

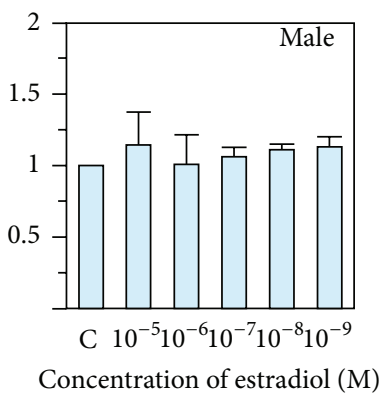

(f)

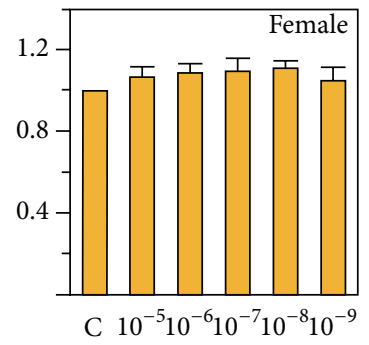

Concentration of testosterone $(\mathrm{M})$

(c)

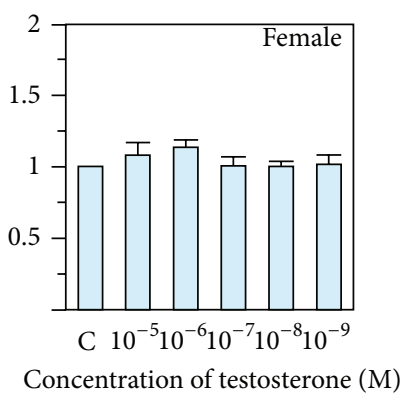

(g)

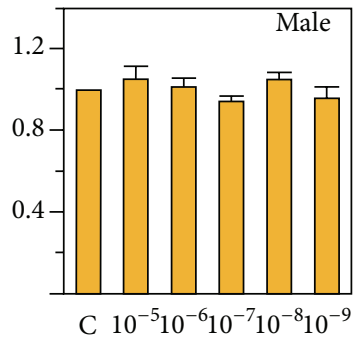

Concentration of testosterone $(\mathrm{M})$

(d)

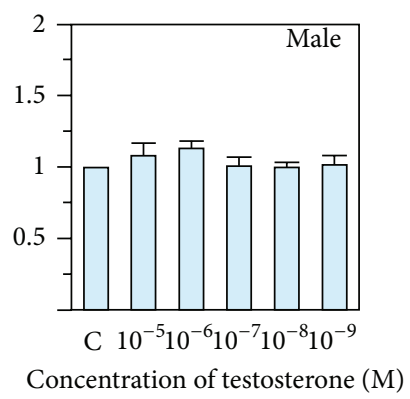

(h)

FIGURE 1: The results of MTT assays and GAG synthesis in articular chondrocytes from female and male rabbits following incubation with $17 \beta$-estradiol or testosterone for 3 days. The experiments were conducted in triplicate, and a mean \pm standard deviation was expressed. Data were normalized to the control values, which were set at $1.0\left({ }^{*} P<0.05,{ }^{* *} P<0.001\right)$.

visualize the nuclei. The stained cells were examined using a fluorescence microscope equipped with a digital camera (Olympus IX-71, Japan).

2.8. Statistics. All of the biochemical data are presented as the mean with $95 \%$ confidence intervals. The data between the different doses of sex hormones were analyzed using one-way independent ANOVA and post hoc Scheffe's tests. The data for the different hormones and genders were analyzed using multiway ANOVA. The statistical analysis was performed using the Statistical Package for the Social Sciences (SPSS version 10.0; SPSS, Chicago, IL). The chosen level of significance was $P<0.05$.

\section{Results and Discussion}

3.1. MTT Assay and GAG Assay. A significant increase in cell proliferation, as indicated by the MTT assay, was observed in the chondrocytes from female rabbits following incubation with $10^{-6}$ and $10^{-7} \mathrm{M} 17 \beta$-estradiol $(P=0.02$ compared to the control) (Figure 1(a)). However, a significant decrease in cell proliferation was observed in male rabbit chondrocytes after incubation with $10^{-5}$ to $10^{-8} \mathrm{M}(P=0.02-0.03) 17 \beta$-estradiol (Figure 1(b)). No significant influence on MTT assay results was observed in chondrocytes incubated with testosterone, regardless of the origin of the cells (Figures 1(c) and 1(d)).

GAG synthesis was significantly increased in female rabbit chondrocytes incubated with $10^{-6}$ to $10^{-8} \mathrm{M} 17 \beta$-estradiol, especially for $10^{-6}$ and $10^{-7} \mathrm{M}(P<0.001)$ (Figure $1(\mathrm{e})$ ). No significant effect on GAG synthesis was demonstrated in the male rabbit chondrocytes after any treatment or in the female ones after testosterone treatment (Figures 1(f), 1(g), and $1(h))$.

\subsection{Real-Time PCR Analysis}

3.2.1. Quantitative Evaluation of Col-I, Col-II, and Aggrecan mRNA Expression following Incubation with Estradiol. Compared to the control, an increase in expression of Col-II and aggrecan mRNAs was observed in female rabbit chondrocytes following incubation with $17 \beta$-estradiol, with particular statistical significance for $10^{-6}$ and $10^{-7} \mathrm{M}(P<0.001)$ (Figures 2(b) and 2(c)). Moreover, the female chondrocytes incubated with any dose of $17 \beta$-estradiol exhibited significantly suppressed expression of Col-I mRNA $(P<0.001)$ (Figure 2(a)). In the chondrocytes from male rabbits, aggrecan mRNA expression increased, but this increase was only statistically significant for $10^{-6} \mathrm{M}(P=0.04)$ (Figure 2(f)). No effect on the expression of Col-I mRNA was observed after incubation with $17 \beta$-estradiol (Figure $2(\mathrm{~d})$ ). The expression of Col-II mRNA increased significantly following incubation with $10^{-6}$ and $10^{-7} \mathrm{M}(P=0.02) 17 \beta$-estradiol (Figure 2(e)). The highest average increases in the expression of Col-II and aggrecan mRNAs were both detected in the chondrocytes from female rabbits incubated with $10^{-7} \mathrm{M} 17 \beta$-estradiol, and the greatest average decrease in the expression of Col-I 


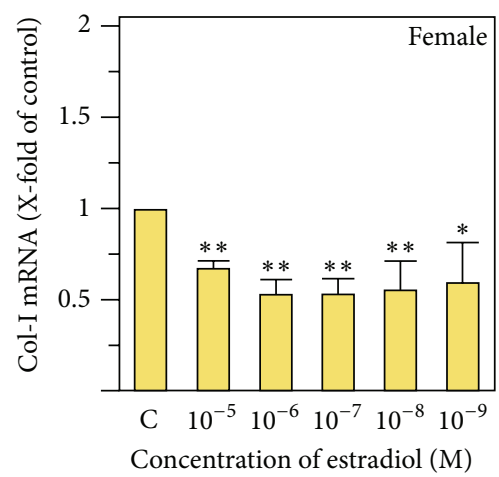

(a)

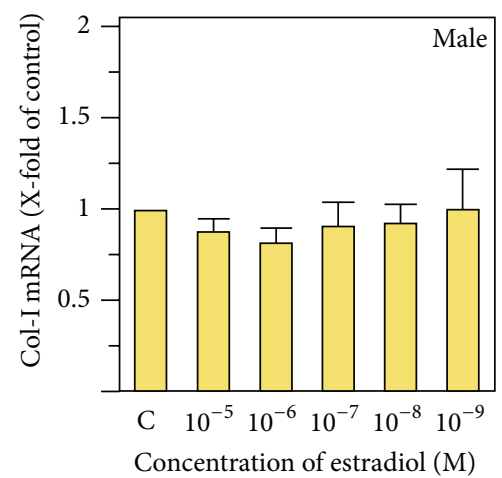

(d)

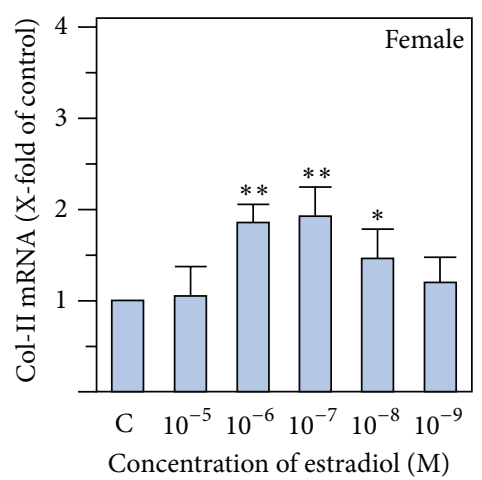

(b)

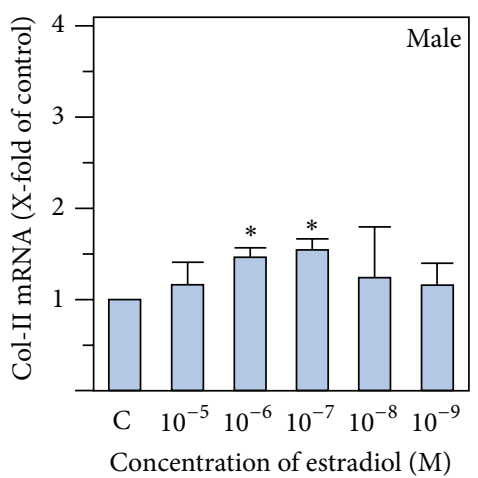

(e)

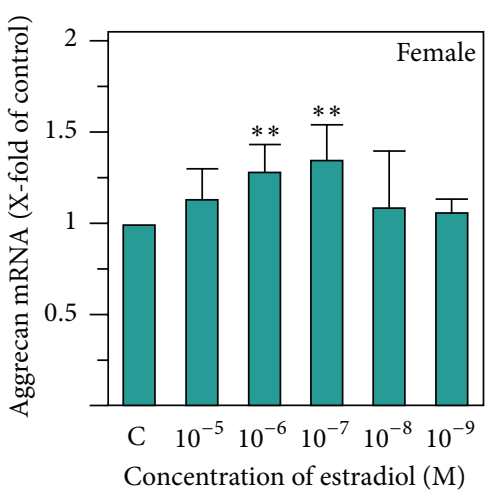

(c)

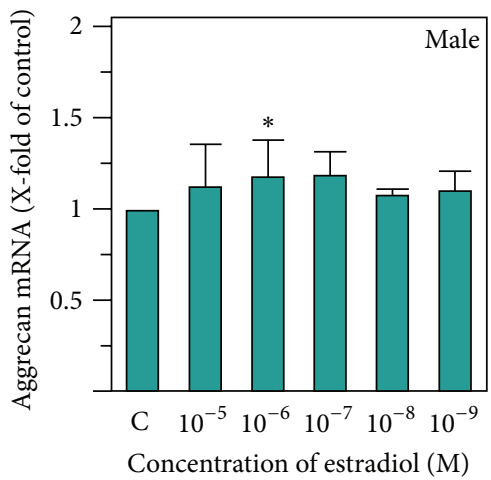

(f)

FIGURE 2: Influence of $17 \beta$-estradiol on the expression of collagen I (Col-I), collagen II (Col-II), and aggrecan mRNA in articular chondrocytes from female and male rabbits for 3 -day culture. The experiments were conducted in triplicate, and a mean \pm standard deviation was expressed. Data were normalized to the control values, which were set at $1.0\left({ }^{*} P<0.05,{ }^{* *} P<0.001\right)$.

TABLE 1: The expression of collagen I and collagen II on different groups with analysis by RT-PCR.

\begin{tabular}{lcccc}
\hline Sex hormone & \multicolumn{2}{c}{$\begin{array}{c}\text { Expression of collagen I (X-fold of control) } \\
\text { Male }\end{array}$} & Female & \multicolumn{2}{c}{$\begin{array}{c}\text { Expression of collagen II (X-fold of control) } \\
\text { Male }\end{array}$} & Female \\
\hline $\begin{array}{c}\text { Estradiol } \\
10^{-6}(n=9)\end{array}$ & $0.77(0.65$ to 0.89$)$ & $0.52^{*}(0.41$ to 0.64$)$ & $1.48(1.39$ to 1.56$)$ & $1.89^{*}(1.76$ to 2.02$)$ \\
$10^{-7}(n=9)$ & $0.89(0.68$ to 1.14$)$ & $0.56^{*}(0.47$ to 0.65$)$ & $1.52(1.39$ to 1.65$)$ & $1.96^{*}(1.72$ to 2.21$)$ \\
Testosterone & & & & \\
$10^{-6}(n=9)$ & $0.98(0.87$ to 1.09$)$ & $0.89(0.78$ to 1.01$)$ & $1.24(0.77$ to 1.71$)$ & $1.31(1.09$ to 1.53$)$ \\
$10^{-7}(n=9)$ & $0.89(0.68$ to 1.10$)$ & $0.78(0.66$ to 0.91$)$ & $1.21(0.74$ to 1.68$)$ & $1.28(1.05$ to 1.51$)$ \\
\hline
\end{tabular}

Values are the mean $(95 \%$ confidence interval $[\mathrm{CI}])$.

$n$ : number of chondrocytes samples.

${ }^{*} P<0.05$ by multiway ANOVA.

mRNA was detected in the chondrocytes from female rabbits incubated with $10^{-6} \mathrm{M} 17 \beta$-estradiol (Table 1).

\subsubsection{Quantitative Evaluation of Col-I, Col-II, and Aggrecan} mRNA Expression following Incubation with Testosterone. In comparison with the control, increased Col-II mRNA expression was observed in the male rabbit chondrocytes after incubation with testosterone. Furthermore, significant effects were observed upon incubation with $10^{-6}$ and $10^{-7} \mathrm{M}$ testosterone in chondrocytes from either male $(P=0.04$ and 0.02 ) or female (both $P=0.02$ ) rabbits (Figures $3(\mathrm{~b})$ and 4(e)). Incubation with different doses of testosterone had no effect on the mRNA expression of Col-I or aggrecan in the chondrocytes from either gender (Figures 3(a), 3(c), 3(d), and $3(f))$.

3.2.3. Quantitative Analysis of TIMP-3 and MMP-3 $m R N A$ Expression following Incubation with Estradiol. TIMP-3 mRNA expression was significantly increased in chondrocytes from female rabbits following incubation with $10^{-5}$ to $10^{-8} \mathrm{M}(P<0.001) 17 \beta$-estradiol and from male rabbits at concentrations of $10^{-5}$ to $10^{-7} \mathrm{M}(P<0.001)$ 


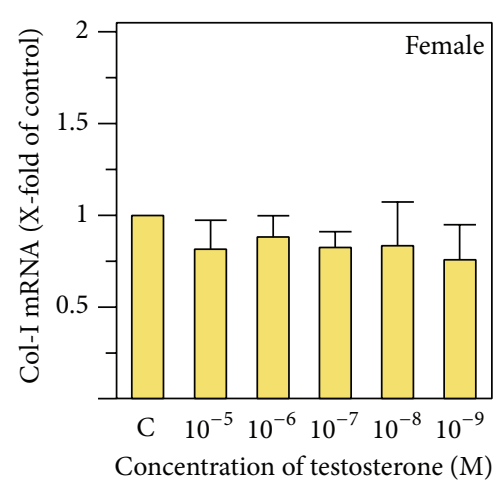

(a)

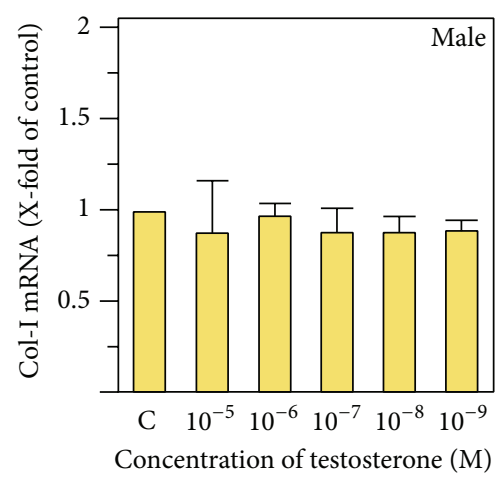

(d)

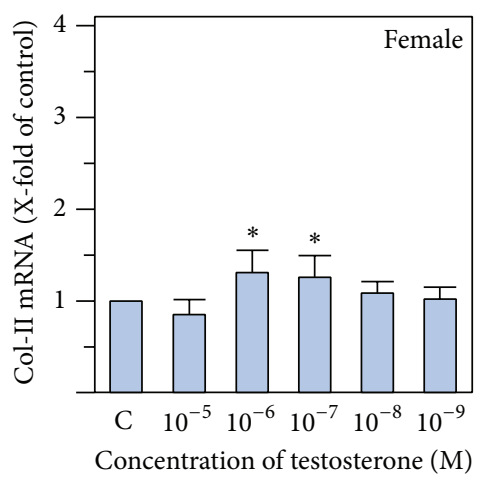

(b)

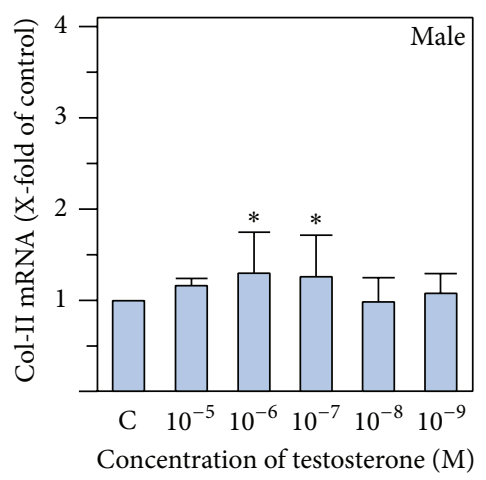

(e)

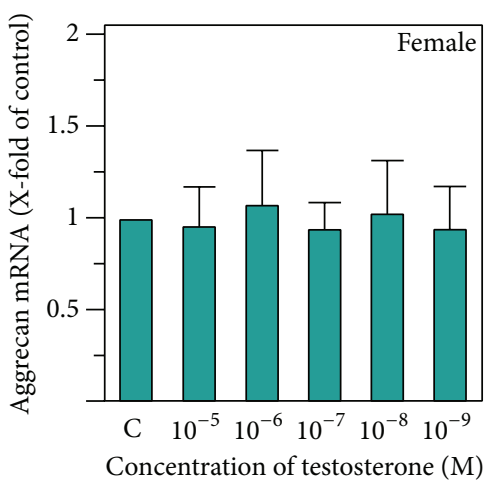

(c)

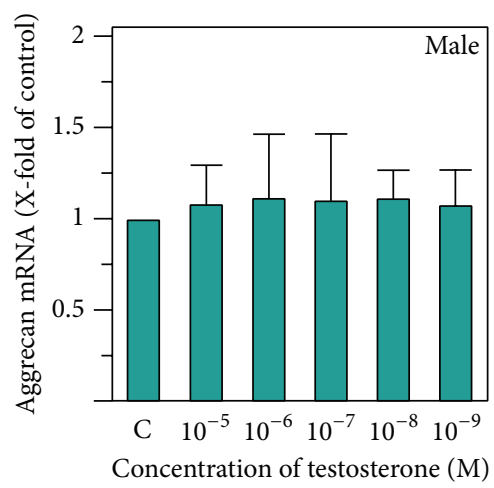

(f)

FIGURE 3: Influence of testosterone on the expression of collagen I (Col-I), collagen II (Col-II), and aggrecan mRNA in articular chondrocytes from female and male rabbits for 3-day culture. The experiments were conducted in triplicate, and a mean \pm standard deviation was expressed. Data were normalized to the control values, which were set at $1.0\left({ }^{*} P<0.05\right)$.

TABLE 2: The expression of TIMP-3 and MMP-3 on different groups with analysis by RT-PCR.

\begin{tabular}{ccccc}
\hline Sex hormone & \multicolumn{2}{c}{$\begin{array}{c}\text { Expression of TIMP-3 (X-fold of control) } \\
\text { Male }\end{array}$} & Female & \multicolumn{2}{c}{$\begin{array}{c}\text { Expression of MMP-3 (X-fold of control) } \\
\text { Male }\end{array}$} \\
\hline $\begin{array}{c}\text { Estradiol } \\
10^{-6}(n=9)\end{array}$ & $2.89(2.68$ to 3.11$)$ & $22.41^{* *}(20.99$ to 23.83$)$ & $0.31(0.23$ to 0.39$)$ & $0.09{ }^{*}(0.07$ to 0.11$)$ \\
$10^{-7}(n=9)$ & $2.77(2.62$ to 2.92$)$ & $13.68^{* *}(9.54$ to 17.42$)$ & $0.24(0.17$ to 0.31$)$ & $0.18(0.13$ to 0.23$)$ \\
Testosterone & & & & \\
$10^{-6}(n=9)$ & $0.72(0.69$ to 0.75$)$ & $3.21(3.11$ to 3.31$)$ & $0.68(0.56$ to 0.81$)$ & $0.17(0.15$ to 0.19$)$ \\
$10^{-7}(n=9)$ & $0.89(0.78$ to 1.01$)$ & $3.31(1.72$ to 4.91$)$ & $0.77(0.50$ to 1.04$)$ & $0.18(0.14$ to 0.22$)$ \\
\hline
\end{tabular}

Values are the mean $(95 \%$ confidence interval $[\mathrm{CI}])$.

$n$ : number of chondrocytes samples.

${ }^{*} P=0.001$ and ${ }^{* *} P<0.001$ by multiway ANOVA.

(Figures 4(a) and 4(b)). Furthermore, the expression of MMP-3 mRNA was significantly suppressed in the chondrocytes from female rabbits following incubation with $10^{-5}$ to $10^{-8} \mathrm{M}(P<0.001) 17 \beta$-estradiol and from male rabbits at doses of $10^{-6}$ and $10^{-7} \mathrm{M}(P<0.05)$ (Figures $4(\mathrm{c})$ and $4(\mathrm{~d})$ ). The greatest increase in the expression of TIMP-3 mRNA and decrease in the expression of MMP-3 mRNA were both detected in the chondrocytes from female rabbits incubated with $10^{-6} \mathrm{M} 17 \beta$-estradiol (Table 2).
3.2.4. Quantitative Evaluation of TIMP-3 and MMP-3 $m R N A$ Expression following Incubation with Testosterone. Compared to the control, the expression of TIMP-3 mRNA was increased and the expression of MMP-3 mRNA was suppressed significantly in female rabbit chondrocytes after incubation with $10^{-5}$ to $10^{-8} \mathrm{M}$ testosterone $(P<0.001)$ (Figures 4(e) and 4(g)). In the chondrocytes from male rabbits, incubation with testosterone had no significant influence on the expression of TIMP-3 mRNA (Figure 4(f)). However, 


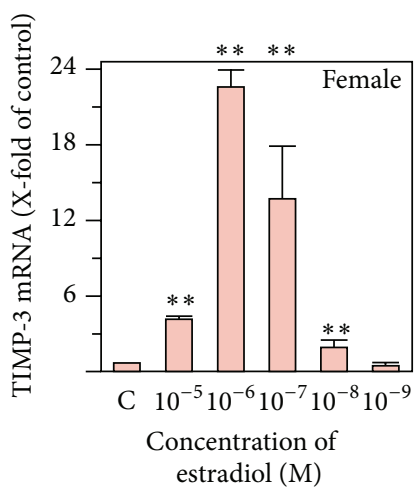

(a)

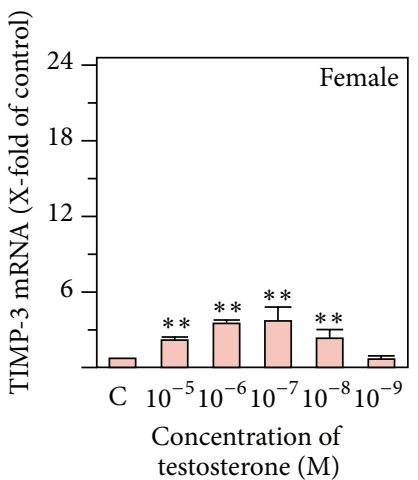

(e)

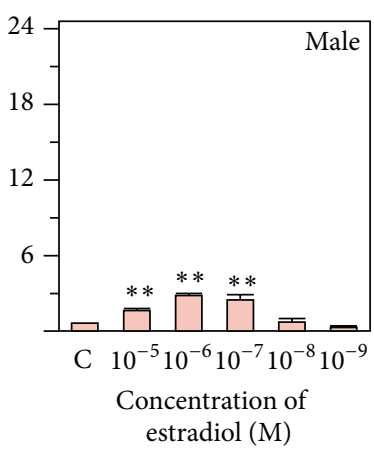

(b)

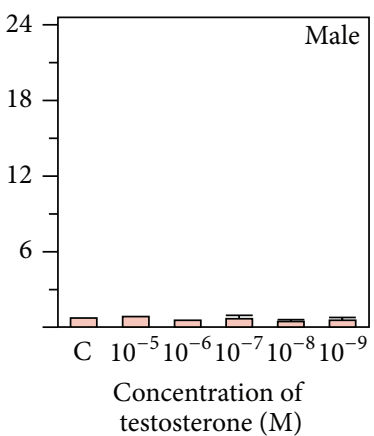

(f)

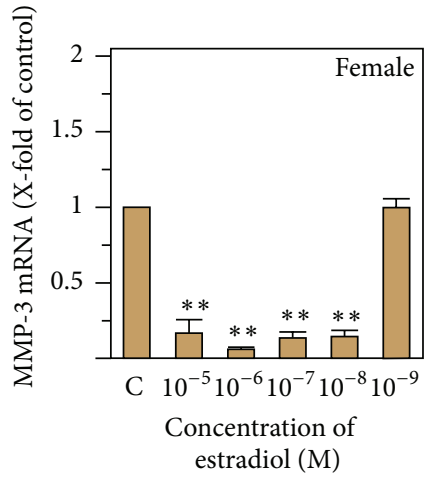

(c)

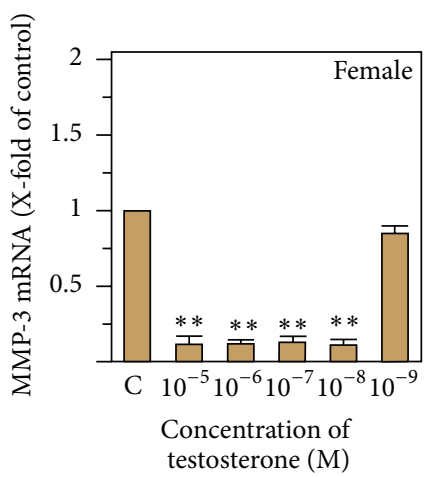

(g)

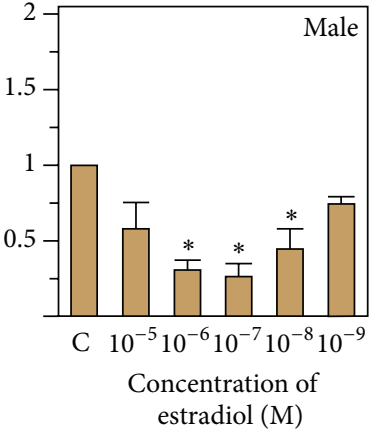

(d)

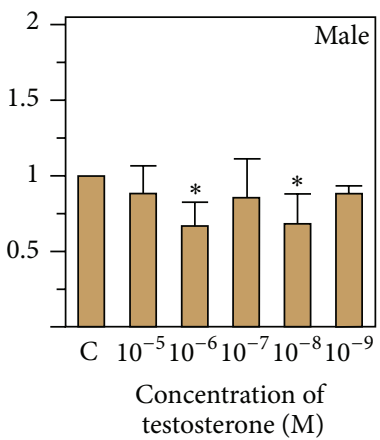

(h)

FIGURE 4: Influence of $17 \beta$-estradiol and testosterone on the expression of tissue inhibitor of matrix metalloproteinase-3 (TIMP-3) and matrix metalloproteinase-3 (MMP-3) mRNAs in articular chondrocytes from female and male rabbits for 3-day culture. The experiments were conducted in triplicate, and a mean \pm standard deviation was expressed. Data were normalized to the control values, which were set at $1.0\left({ }^{*} P<0.05,{ }^{* *} P<0.001\right)$.

a trend toward decreased MMP-3 mRNA expression was observed in male rabbit chondrocytes following incubation with testosterone, but this difference was only significant at the concentrations of $10^{-6}$ and $10^{-8} \mathrm{M}(P=0.03)$ (Figure 4(h)).

3.2.5. Immunocytochemical Staining. Positive reactions with antibodies against Col-II were observed in groups of control, cells incubated with $10^{-6} \mathrm{M} 17 \beta$-estradiol or testosterone. However, greater staining for collagen II expression was presented in the chondrocytes incubated with $10^{-6} \mathrm{M} 17 \beta$ estradiol than in control cells or cells incubated with testosterone (Figure 5).

In our MTT assays, all doses $\left(10^{-5}\right.$ to $\left.10^{-9} \mathrm{M}\right)$ of $17 \beta$ estradiol resulted in increased proliferation in the female rabbit chondrocytes, although significant increases were found only at the doses of $10^{-6}$ and $10^{-7} \mathrm{M}$. In addition, a significant increase in the expression of aggrecan mRNA was observed at doses of $10^{-6}$ and $10^{-7} \mathrm{M} 17 \beta$-estradiol (Figure $2(\mathrm{c})$ ). These results can explain why female rabbit chondrocytes stimulated with $10^{-6}$ and $10^{-7} \mathrm{M} 17 \beta$-estradiol exhibited the highest levels of GAG synthesis (Figure 1(e)). However, supraphysiological doses of $17 \beta$-estradiol $\left(10^{-5}\right.$ to $\left.10^{-8} \mathrm{M}\right)$ led to significantly lower proliferation in the male rabbit chondrocytes, whereas the physiological dose $\left(10^{-9} \mathrm{M}\right)$ had no effect. In contrast, no significant influence was observed based on the results of cell proliferation or GAG analysis after incubation with testosterone either in the female or in the male chondrocytes. Furthermore, no significant influence of testosterone on aggrecan gene expression was observed in both genders (Figures 3(c) and 3(f)), which corresponded to the GAG assay result (Figures $1(\mathrm{~g})$ and $1(\mathrm{~h})$ ).

In OA cartilage, Col-II manifested a decreased staining and Col-I was found as a thin layer covering the surface of fibrillated cartilage [16]. Positive effects on the articular chondrocytes, including the upregulation of Col-II and aggrecan or downregulation of Col-I expression, were observed following incubation with $17 \beta$-estradiol in female chondrocytes. In contrast, an increase in only Col-II mRNA expression was observed at $10^{-6}$ and $10^{-7} \mathrm{M}$ of testosterone in both genders (Figures 3(b) and 3(e)), with no significant difference between the genders (Table 1). In the comparison of different genders and sex hormones, the stimulation of Col-II mRNA expression and suppression of Col-I mRNA expression were significantly greater in the female cells with estradiol than in the male cells with testosterone (Table 2). In addition to Col-I, Col-II, and aggrecan, MMPs and TIMPs also play 


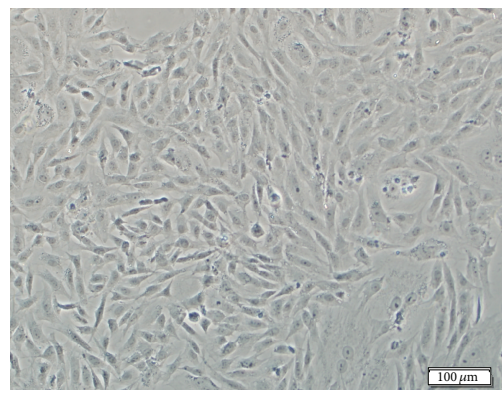

(a)

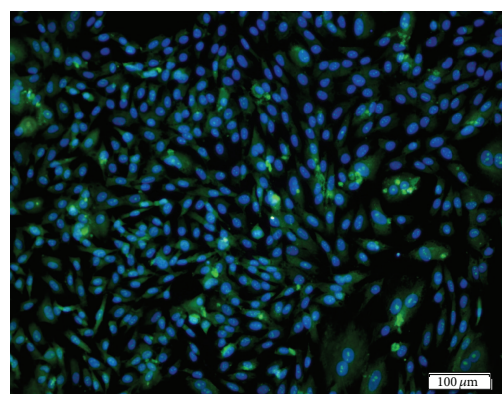

(d)

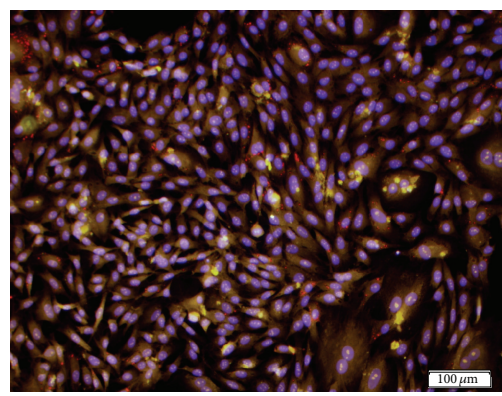

(g)

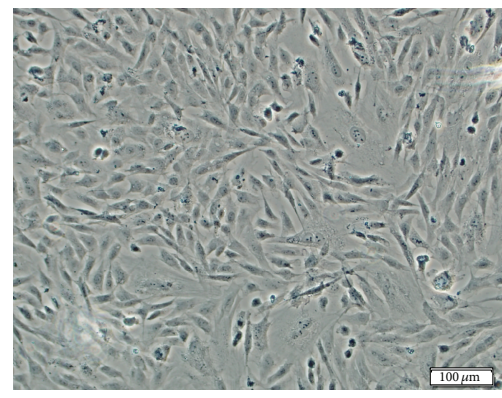

(b)

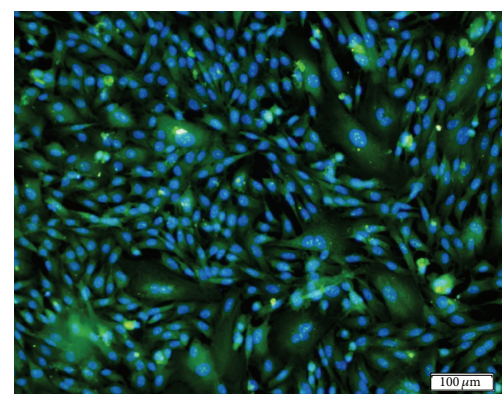

(e)

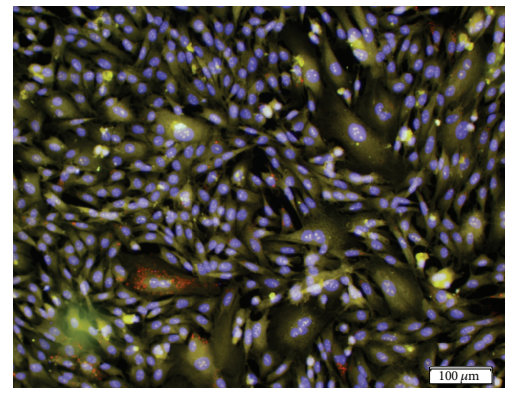

(h)

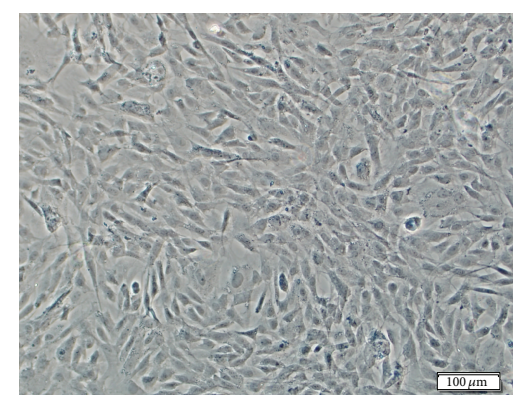

(c)

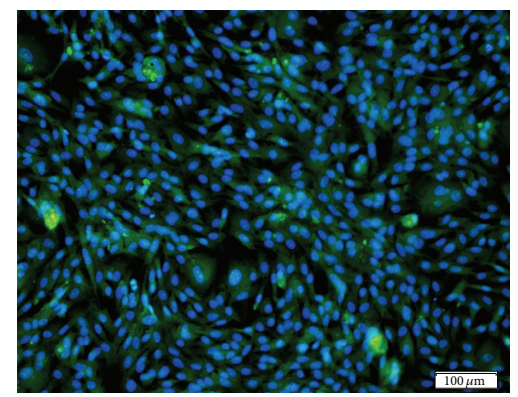

(f)

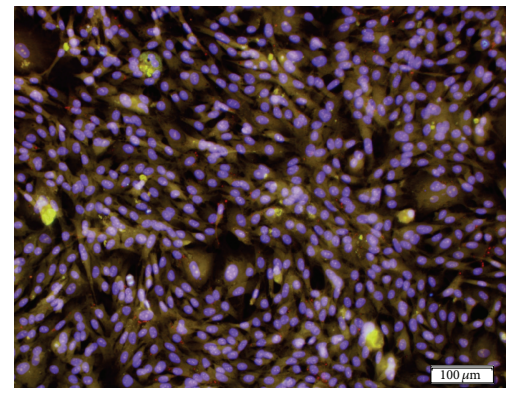

(i)

FIGURE 5: Immunocytochemical staining in female rabbit chondrocytes following incubation with sex hormones for 3 days. Optic microscopic view of (a) controls, (b) cells incubated with $10^{-6} \mathrm{M} 17 \beta$-estradiol, and (c) cells incubated with $10^{-6} \mathrm{M}$ testosterone. Immunocytochemical detection of Col-II of (d) controls, (e) cells incubated with $10^{-6} \mathrm{M} 17 \beta$-estradiol, and (f) cells incubated with $10^{-6} \mathrm{M}$ testosterone and immunocytochemical staining for combination of Col-I and Col-II in (g) controls, (h) cells incubated with $10^{-6} \mathrm{M} 17 \beta$-estradiol, and (i) cells incubated with $10^{-6} \mathrm{M}$ testosterone. All showed positive immunoreactivity for Col-II, and stronger positive staining was observed in the estradiol group.

important roles in OA. MMPs degrade ECM molecules, including cartilage proteoglycan and collagen II, whereas TIMPs inhibit MMPs by forming high-affinity complexes. Several studies have demonstrated that, in the pathogenesis of OA, MMPs may be overexpressed relative to TIMPs $[17,18]$. The increased expression of MMPs will degrade Col-II and aggrecan, whereas the expression of TIMPs will prevent the degradation of articular cartilage. In our experiments, the highest levels of TIMP-3 mRNA expression were observed in female chondrocytes after incubation with $10^{-6}$ and $10^{-7} \mathrm{M}$ estradiol, and the lowest expression of MMP-3 mRNA was observed in female chondrocytes after incubation with $10^{-6} \mathrm{M}$ estradiol. These results likely reflect the fact that GAG synthesis was the highest in the female chondrocytes at $10^{-6}$ and $10^{-7} \mathrm{M}$ estradiol. Song et al. demonstrated that increased MMP-3 mRNA in the knee cartilage of ovariectomized rabbits could be suppressed by estrogen supplementation [19]. Similarly, our results indicated that supraphysiological doses $\left(10^{-5}\right.$ to $\left.10^{-8} \mathrm{M}\right)$ of $17 \beta$-estradiol could significantly suppress the expression of MMP-3 mRNA. Claassen et al. also showed significant suppressing effect of incubation with $10^{-5} \mathrm{M}$ of $17 \beta$-estradiol on MMPs mRNA expression in female chondrocytes [20]. The gene expression results indicated that $17 \beta$-estradiol affected Col-I and ColII in female rabbit chondrocytes, significantly promoting the expression of Col-II mRNA and suppressing the expression of Col-I. The concentrations of $10^{-6}$ and $10^{-7} \mathrm{M}$ induced the most balanced collagen expression in articular cartilage. Furthermore, incubation of the female rabbit chondrocytes with $10^{-6} \mathrm{M} 17 \beta$-estradiol produced the highest expression of TIMP-3 mRNA and the lowest expression of MMP-3 mRNA between the different sex hormones and genders. 
According to RT-PCR, MTT, and GAG synthesis assays using female rabbit chondrocytes incubated with $17 \beta$ estradiol, the ideal concentrations for a positive effect on cartilage were $10^{-6}$ and $10^{-7} \mathrm{M}$, which are supraphysiological doses. However, the positive effect was reduced at a dose of $10^{-5} \mathrm{M}$ versus $10^{-6}$ and $10^{-7} \mathrm{M}$. The higher concentrations are potentially toxic to chondrocytes. Previous studies demonstrated that $17 \beta$-estradiol applied at $10^{-4}$ to $10^{-5} \mathrm{M}$ caused a significant loss of GAG in the fibrocartilage of the pubic symphysis in ovariectomized rabbits [21,22]. No significant effect was observed for the physiological dose of estradiol in our studies. Claassen et al. also reported that incubation with physiological dose of estradiol alone did not significantly influence proline incorporation or collagen II synthesis in their study of cow chondrocytes [23].

Col-II is the main structural component of the ECM of articular cartilage. Col-I is not expressed in healthy articular cartilage, but it can be detected in degenerated cartilage or fibrocartilage. In our study, when chondrocytes were cultured in two-dimensional culture, their tendency to dedifferentiate was accompanied by a switch from collagen II to collagen I synthesis. Both Col-I and Col-II were observed via immunocytochemical staining, and a stronger immunoreaction for Col-II was observed in $10^{-6} \mathrm{M} 17 \beta$ estradiol-treated cells than in testosterone-treated or control cells. The immunocytochemistry findings were consistent with a collagen-stimulating effect for sex hormones, which both stimulated Col-II mRNA expression and increased the volume of Col-II in the ECM. A stronger regulation was observed for $17 \beta$-estradiol than for testosterone.

\section{Conclusions}

The findings of our study suggest that $17 \beta$-estradiol plays a more important role than testosterone in the biological functions and gene expression profiles of chondrocytes, especially in female chondrocytes. Furthermore, both protective and stimulating effects of $17 \beta$-estradiol on female chondrocytes were observed with the greatest effects at $10^{-6}$ and $10^{-7} \mathrm{M}$ in two-dimensional culture. Therefore, further studies are needed to test the effect of $10^{-6}$ or $10^{-7} \mathrm{M} 17 \beta$-estradiol in chondrocytes using three-dimensional culture or in vivo animal studies.

\section{Conflict of Interests}

The authors declare that there is no conflict of interests regarding the publication of this paper.

\section{Authors' Contribution}

Shwu Jen Chang and Shyh Ming Kuo contributed equally to the present work.

\section{Acknowledgment}

This study was financially supported by a Grant from the National Science Council, Taiwan (NSC 98-2221-E-214-010MY3).

\section{References}

[1] A. E. Wluka, F. M. Cicuttini, and T. D. Spector, "Menopause, oestrogens and arthritis," Maturitas, vol. 35, no. 3, pp. 183-199, 2000.

[2] V. K. Srikanth, J. L. Fryer, G. Zhai, T. M. Winzenberg, D. Hosmer, and G. Jones, "A meta-analysis of sex differences prevalence, incidence and severity of osteoarthritis," Osteoarthritis and Cartilage, vol. 13, no. 9, pp. 769-781, 2005.

[3] J. A. Gokhale, S. R. Frenkel, and P. E. Dicesare, "Estrogen and osteoarthritis," The American Journal of Orthopedics, vol. 33, no. 2, pp. 71-80, 2004

[4] H.-L. Ma, T. J. Blanchet, D. Peluso, B. Hopkins, E. A. Morris, and S. S. Glasson, "Osteoarthritis severity is sex dependent in a surgical mouse model," Osteoarthritis and Cartilage, vol. 15, no. 6, pp. 695-700, 2007.

[5] D. von Mühlen, D. Morton, C. A. von Mühlen, and E. BarrettConnor, "Postmenopausal estrogen and increased risk of clinical osteoarthritis at the hip, hand, and knee in older women," Journal of Women's Health and Gender-Based Medicine, vol. 11, no. 6, pp. 511-518, 2002.

[6] K. P. Ng, J. P. Datuin, and H. A. Bern, "Effects of estrogens in vitro and in vivo on cartilage growth in the tilapia (Oreochromis mossambicus)," General and Comparative Endocrinology, vol. 121, no. 3, pp. 295-304, 2001.

[7] H. Claassen, M. Schünke, and B. Kurz, "Estradiol protects cultured articular chondrocytes from oxygen-radical-induced damage," Cell and Tissue Research, vol. 319, no. 3, pp. 439-445, 2005.

[8] P. Høegh-Andersen, L. B. Tankó, T. L. Andersen et al., "Ovariectomized rats as a model of postmenopausal osteoarthritis: validation and application," Arthritis Research \& Therapy, vol. 6, no. 2, pp. R169-180, 2004.

[9] H. Claassen, C. Cellarius, K. E. Scholz-Ahrens et al., "Extracellular matrix changes in knee joint cartilage following bone-active drug treatment," Cell and Tissue Research, vol. 324, no. 2, pp. 279-289, 2006.

[10] E. Nasatzky, Z. Schwartz, B. D. Boyan, W. A. Soskolne, and A. Ornoy, "Sex-dependent effects of 17-beta-estradiol on chondrocyte differentiation in culture," Journal of Cellular Physiology, vol. 154, no. 2, pp. 359-367, 1993.

[11] T. Irie, T. Aizawa, and S. Kokubun, "The role of sex hormones in the kinetics of chondrocytes in the growth plate. A study in the rabbit," Journal of Bone and Joint Surgery B, vol. 87, no. 9, pp. 1278-1284, 2005.

[12] D. D. Dean, "Proteinase-mediated cartilage degradation in osteoarthritis," Seminars in Arthritis and Rheumatism, vol. 20, no. 6, pp. 2-11, 1991.

[13] T. D. Schmittgen and K. J. Livak, "Analyzing real-time PCR data by the comparative CT method," Nature Protocols, vol. 3, no. 6, pp. 1101-1108, 2008.

[14] A. B. Khoshfetrat, M. Kino-oka, Y. Takezawa, T. Yamamoto, K. Sugawara, and M. Taya, "Seeding density modulates migration and morphology of rabbit chondrocytes cultured in collagen gels," Biotechnology and Bioengineering, vol. 102, no. 1, pp. 294302, 2009.

[15] R. R. Varshney, R. Zhou, J. Hao et al., "Chondrogenesis of synovium-derived mesenchymal stem cells in gene-transferred co-culture system," Biomaterials, vol. 31, no. 26, pp. 6876-6891, 2010.

[16] D. Pfander, R. Rahmanzadeh, and E. E. Scheller, "Presence and distribution of collagen II, collagen I, fibronectin, and 
tenascin in rabbit normal and osteoarthritic cartilage," Journal of Rheumatology, vol. 26, no. 2, pp. 386-394, 1999.

[17] R. A. Greenwald, "Treatment of destructive arthritic disorders with MMP inhibitors. Potential role of tetracyclines," Annals of the New York Academy of Sciences, vol. 732, pp. 181-198, 1994.

[18] J. Martel-Pelletier, R. McCollum, N. Fujimoto, K. Obata, J. M. Cloutier, and J.-P. Pelletier, "Excess of metalloproteases over tissue inhibitor of metalloprotease may contribute to cartilage degradation in osteoarthritis and rheumatoid arthritis," Laboratory Investigation, vol. 70, no. 6, pp. 807-815, 1994.

[19] Y.-J. Song, Z.-H. Wu, S.-Q. Lin, X.-S. Weng, and G.-X. Qiu, "The effect of estrogen and progestin on the expression of matrix metalloproteinases, tissue inhibitor of metalloproteinase and interleukin-lbeta mRNA in synovia of OA rabbit model," Zhonghua Yi Xue Za Zhi, vol. 83, no. 6, pp. 498-503, 2003.

[20] H. Claassen, R. Steffen, J. Hassenpflug et al., "17 $\beta$-estradiol reduces expression of MMP-1, -3 , and -13 in human primary articular chondrocytes from female patients cultured in a three dimensional alginate system," Cell and Tissue Research, vol. 342, no. 2, pp. 283-293, 2010.

[21] H. Claassen, V. Briese, F. Manapov, B. Nebe, M. Schünke, and B. Kurz, "The phytoestrogens daidzein and genistein enhance the insulin-stimulated sulfate uptake in articular chondrocytes," Cell and Tissue Research, vol. 333, no. 1, pp. 71-79, 2008.

[22] G. Hashem, Q. Zhang, T. Hayami, J. Chen, W. Wang, and S. Kapila, "Relaxin and $\beta$-estradiol modulate targeted matrix degradation in specific synovial joint fibrocartilages: progesterone prevents matrix loss," Arthritis Research and Therapy, vol. 8, no. 4, article R98, 2006.

[23] H. Claassen, M. Schlüter, M. Schünke, and B. Kurz, "Influence of $17 \beta$-estradiol and insulin on type II collagen and protein synthesis of articular chondrocytes," Bone, vol. 39, no. 2, pp. 310$317,2006$. 


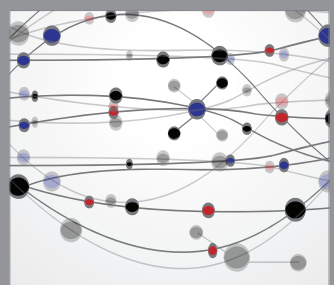

The Scientific World Journal
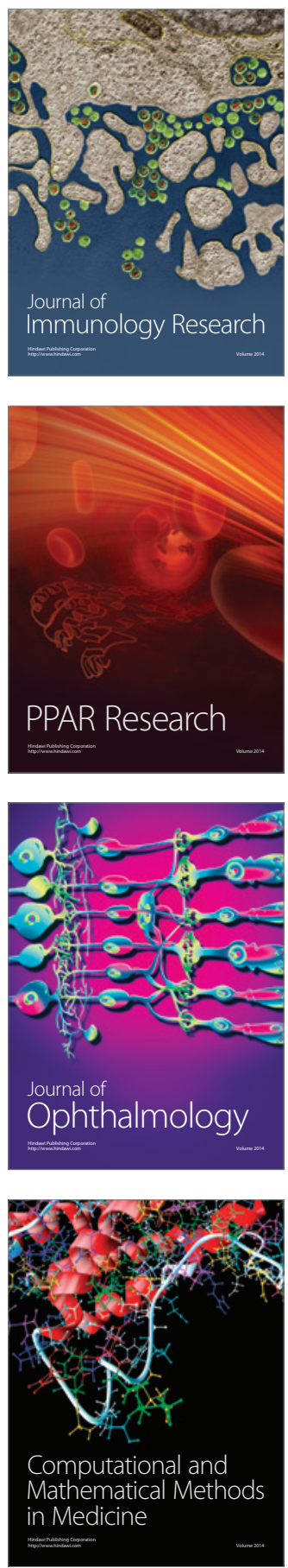

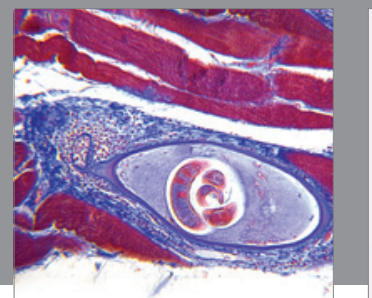

Gastroenterology

Research and Practice
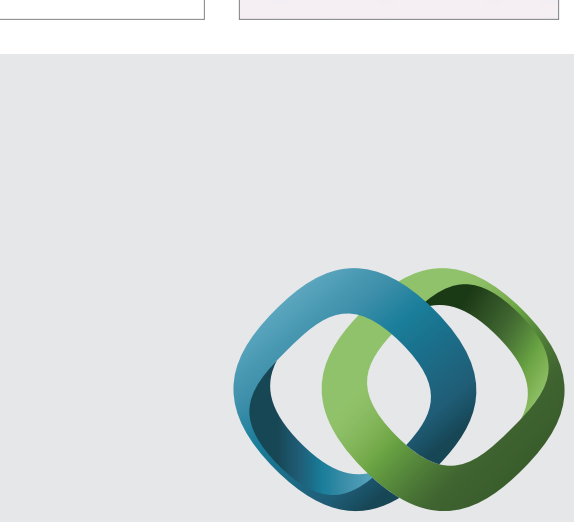

\section{Hindawi}

Submit your manuscripts at

http://www.hindawi.com
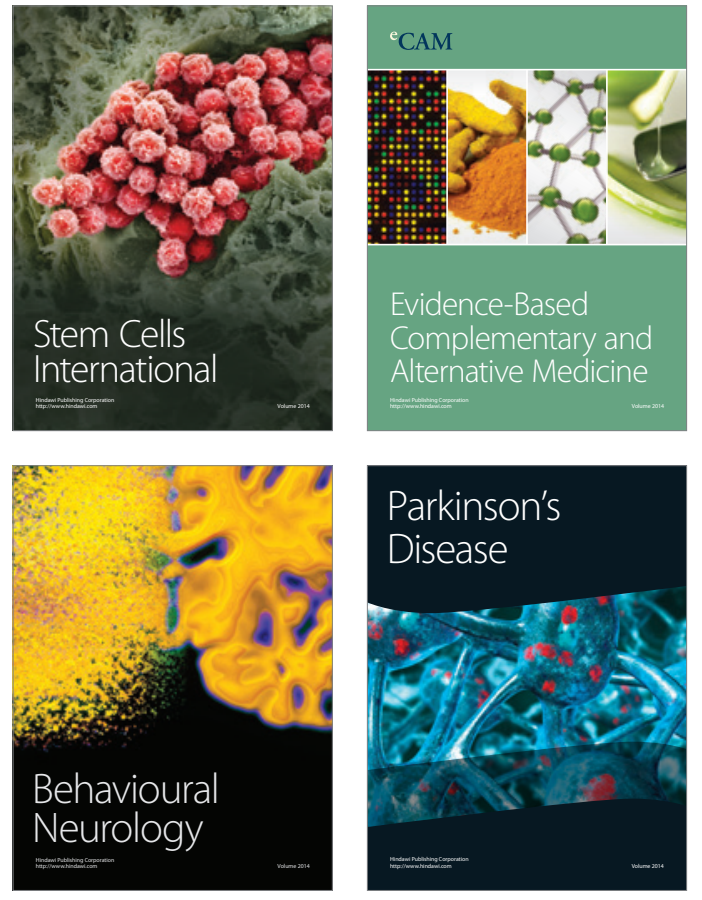
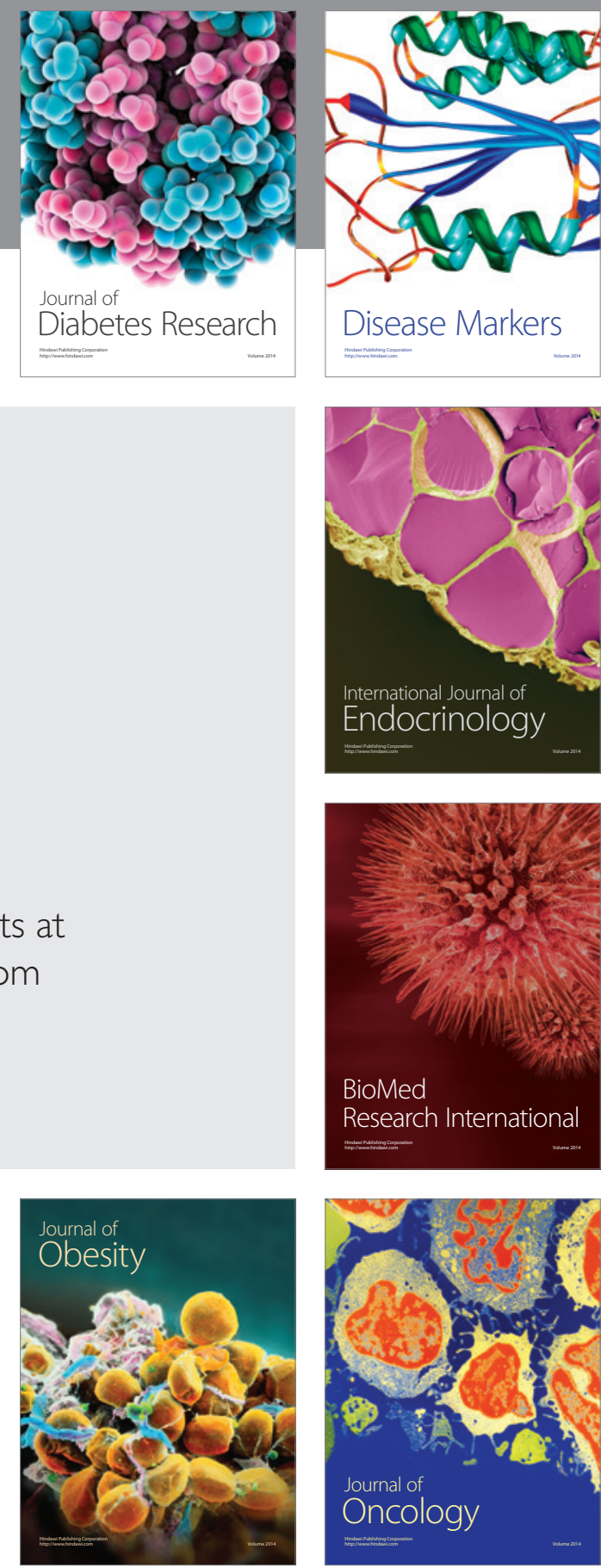

Disease Markers
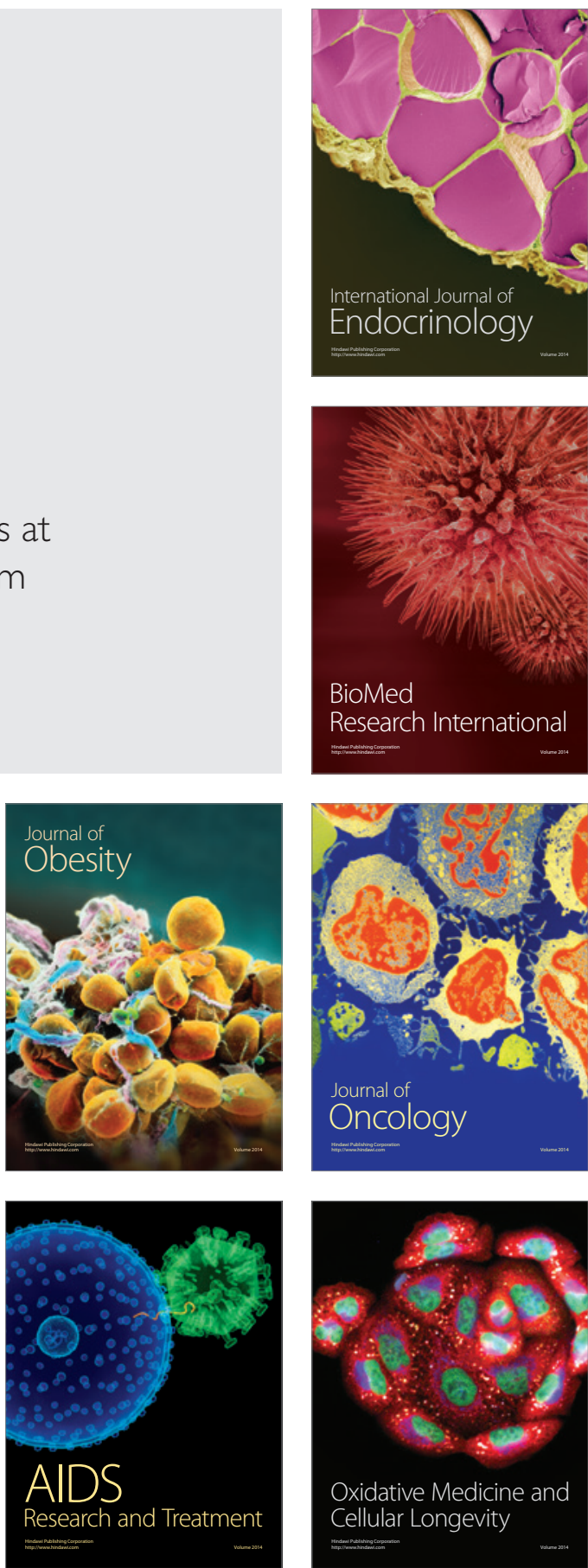